\title{
Spatiotemporal Scatter Models for Dynamic SPECT
}

\author{
Bryan W. Reutter, Senior Member, IEEE, Grant T. Gullberg, Fellow, IEEE, \\ and Ronald H. Huesman, Fellow, IEEE
}

\begin{abstract}
Dynamic single photon emission computed tomography (SPECT) data acquisition and quantitative kinetic data analysis provide unique information that can enable improved discrimination between healthy and diseased tissue, compared to conventional static imaging. Previously, we modeled time courses of activity within segmented SPECT volumes of interest and developed algorithms to estimate kinetic model parameters directly from dynamic projection data. We now propose two methods for modeling and estimating scatter jointly with tracer kinetic models. The goal is to reduce bias in kinetic parameter estimates by properly accounting for scatter. These methods exploit the fact that the scatter distribution from a volume of interest is spatially smooth and has the same temporal kinetics as unscattered events from the volume. The first method treats scattered events as if they originate from scatter sites distributed in image space. For each volume of interest, the distribution of scatter sites is modeled with a smooth spatial function and events from this effective scatter source distribution (ESSD) are forward-projected along with unscattered events from the volume. Thus, the projector only needs to model non-scatter effects. The second method bypasses modeling an ESSD in image space and simply models the spatial projection of scatter to be a smooth function in projection space. Computer simulations of a dynamic ${ }^{99 m}$ Tc-teboroxime cardiac SPECT scan show that unscattered and scattered events from the blood pool, myocardium, and liver have distinct spatiotemporal signatures and that it is feasible to jointly estimate scatter amplitudes and time-activity curves for volumes of interest directly from projection data. This suggests that joint estimation of scatter, blood input function, and compartmental model parameters is a well-posed problem and can lead to reduced bias in kinetic parameter estimates.
\end{abstract}

\section{INTRODUCTION}

D YNAMIC single photon emission computed tomography (SPECT) data acquisition and quantitative kinetic data analysis provide unique information that can enable improved discrimination between healthy and diseased tissue, compared to conventional static imaging [1], [2]. For example, in a dynamic cardiac study one can identify unwanted scatter from the liver because the scattered liver activity has the same temporal kinetics as unscattered liver activity. In this work, we present methods for modeling and estimating scatter jointly with tracer kinetic models. The goal is to reduce bias in kinetic

This work was supported by the U.S. Department of Health and Human Services under grants R01-HL50663 and R01-EB00121 and by the Director, Office of Science, Office of Biological and Environmental Research, Medical Sciences Division of the U.S. Department of Energy under contract DE-AC0376SF00098. This work was developed in part with the use of resources at the U.S. Department of Energy National Energy Research Scientific Computing (NERSC) Center.

The authors are with the Department of Functional Imaging, Lawrence Berkeley National Laboratory, University of California, Berkeley, CA 94720, USA (e-mail: bwreutter@lbl.gov). parameter estimates by properly accounting for scatter [3]. These methods exploit the fact that the scatter distribution from a volume of interest is spatially smooth and has the same temporal kinetics as unscattered activity from the volume.

\section{Spatiotemporal Models}

Previously, we modeled time courses of radiotracer activity within segmented SPECT volumes of interest, and developed algorithms to estimate kinetic model parameters and their statistical uncertainties directly from dynamic cardiac SPECT projection data [4]-[7]. The time-activity curve for segmented volume $m$ is denoted by $A^{m}(t)$ and can be modeled with use of a compartmental model or temporal B-spline basis functions. The detected count rate at time $t$ for detector element $i, P_{i}(t)$, is modeled as

$$
P_{i}(t)=\sum_{m=1}^{M} U_{i}^{m}(t) A^{m}(t),
$$

where $U_{i}^{m}(t)$ is the spatial projection of a unit concentration of static activity in volume $m$, and $M$ is the number of segmented volumes encompassing the projected field of view. The spatial projection function, $U_{i}^{m}(t)$, models physical effects such as attenuation, depth-dependent point response, and scatter, and is time-varying as a result of gantry motion. For a periodic (e.g., multi-rotation circular) orbit with period $T$, one has $U_{i}^{m}(t+T)=U_{i}^{m}(t)$.

\section{A. Modeling Scatter in the Projector}

If one has an attenuation map and a projector that models scatter (and other physical effects), then spatial projection functions, $U_{i}^{m}(t)$, can be calculated and time-activity curves, $A^{m}(t)$, can be estimated directly from projection data with use of (1). Generally, it is computationally intensive to calculate spatial projection functions that contain scatter as determined by Monte Carlo simulation [8] or analytic ray-driven algorithms [9].

As an alternative, we propose two simpler methods that model and estimate scatter based on the fact that the scatter distribution from a volume of interest is spatially smooth and has the same temporal kinetics as unscattered activity from the volume.

\section{B. Modeling Smoothness of Scatter in Image Space}

The first proposed method builds on work by Frey and Tsui [10], in which scattered events are treated as if they 
originate from scatter sites distributed in image space. For each segmented volume of interest, we model the distribution of scatter sites with a smooth spatial function, and forward-project events from this effective scatter source distribution (ESSD) along with unscattered events from the volume of interest. Thus, the projection operator (denoted below by $\mathcal{F}_{i}$ ) only needs to model other physical effects such as attenuation and depthdependent point response.

The dynamic projection data model (1) can be rewritten as

$$
P_{i}(t)=\sum_{m=1}^{M}\left[D_{i}^{m}(t)+S_{i}^{m}(t)\right] A^{m}(t),
$$

where $D_{i}^{m}(t)$ and $S_{i}^{m}(t)$ are spatial projections of unscattered and scattered static activity, respectively. Time variation of $D_{i}^{m}(t)$ and $S_{i}^{m}(t)$ is due only to gantry motion; dynamics of tracer uptake and washout are modeled by $A^{m}(t)$.

The projection of unscattered events can be written as the spatial integration

$$
D_{i}^{m}(t)=\int_{\mathcal{X}} \mathcal{F}_{i}(\mathbf{x}, t) \mathcal{D}^{m}(\mathbf{x}) d \mathbf{x},
$$

where $\mathbf{x}$ is a point in image space $\mathcal{X}, \mathcal{D}^{m}(\mathbf{x})$ is the indicator function for volume $m$, and $\mathcal{F}_{i}(\mathbf{x}, t)$ is the projection operator that maps image space to detector element $i$ for the gantry position at time $t$.

The spatial projection of scattered events is modeled as

$$
S_{i}^{m}(t)=\int_{\mathcal{X}} \mathcal{F}_{i}(\mathbf{x}, t)\left[\sum_{q=1}^{Q^{m}} b_{q m} \mathcal{B}^{q m}(\mathbf{x})\right] \sigma_{i}^{m}(\mathbf{x}, t) d \mathbf{x},
$$

where $\mathcal{B}^{q m}(\mathbf{x})$ are spatial basis functions that model the ESSD for volume $m, b_{q m}$ are linear coefficients, and $Q^{m}$ is the number of basis functions. The relative fraction of events that originate in volume $m$ and scatter from point $\mathbf{x}$ in the direction of detector element $i$ is denoted by $\sigma_{i}^{m}(\mathbf{x}, t)$, which can be calculated with use of the Klein-Nishina formula [11]. Rearranging (4) yields

$$
S_{i}^{m}(t)=\sum_{q=1}^{Q^{m}} b_{q m} B_{i}^{q m}(t)
$$

where $B_{i}^{q m}(t)=\int_{\mathcal{X}} \mathcal{F}_{i}(\mathbf{x}, t) \mathcal{B}^{q m}(\mathbf{x}) \sigma_{i}^{m}(\mathbf{x}, t) d \mathbf{x}$.

Thus, the spatial projection of scattered events can be modeled as a linear combination of projections of smooth functions. We anticipate that nonuniform rational B-splines (NURBS) [12] will be suitable basis functions for modeling the somewhat peaked centers and long tails of the ESSD.

\section{Modeling Smoothness of Scatter in Projection Space}

The second proposed method bypasses modeling an ESSD in image space and simply models the spatial projection of scatter to be a linear combination of smooth functions in projection space:

$$
S_{i}^{m}(t)=\sum_{r=1}^{R^{m}} c_{r m} C_{i}^{r m}(t)
$$

where $C_{i}^{r m}(t)$ are the basis functions (e.g., NURBS), $c_{r m}$ are linear coefficients, and $R^{m}$ is the number of basis functions.

\section{Computer Simulations}

Computer simulations were performed to test the feasibility of jointly estimating scatter amplitudes and time-activity curves for volumes of interest directly from projection data. Simulated spatial distributions were obtained with use of the idealized single-slice emission phantom shown in Fig. 1. The phantom contained $M=4$ circular volumes of interest: blood pool, left ventricular myocardium, liver, and background tissue. Each volume contained spatially uniform activity.

Fig. 2 shows simulated time-activity curves, $A^{m}(t)$, which mimicked the kinetics of ${ }^{99 \mathrm{~m}}$ Tc-teboroxime [13], [2]. The blood curve was used as the input function for one-compartment models that generated curves for the myocardium and liver. The background tissue curve was proportional to the blood curve.

The simulated 15 min data acquisition with a single-detector system consisted of one $360^{\circ}$ rotation per minute, 120 projection angles per rotation, $I=50$ parallel projection rays per angle, and $L=1800$ time intervals. Projection bins were $6 \mathrm{~mm}$ $\times 6 \mathrm{~mm}$. Uniform attenuation and scatter at $140 \mathrm{keV}$ were simulated with use of a ray-driven projector and analytic line integrals [14], [15], [9]. Depth-dependent collimator response was not simulated. The blood input amplitude was adjusted so that $1 \mathrm{M}, 500 \mathrm{k}$, or $250 \mathrm{k}$ events were detected.

Time-activity curves were estimated directly from projection data with use of the temporal model

$$
A^{m}(t)=\sum_{n=1}^{N} a_{m n} V^{n}(t),
$$

where $V^{n}(t)$ are quadratic B-spline temporal basis functions (Fig. 3), $a_{m n}$ are model coefficients for volume of interest $m$, and $N=16$ is the number of basis functions.

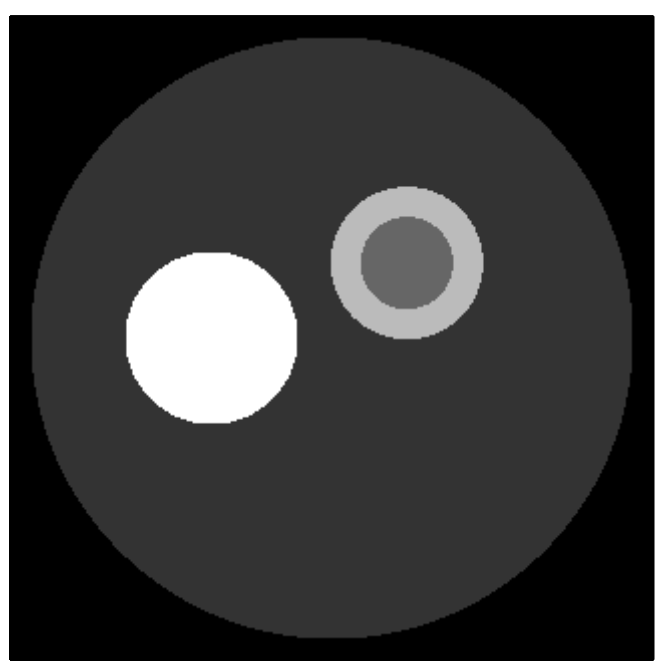

Fig. 1. Emission phantom composed of liver (bright circle), left ventricular myocardium, blood pool, and background tissue. 


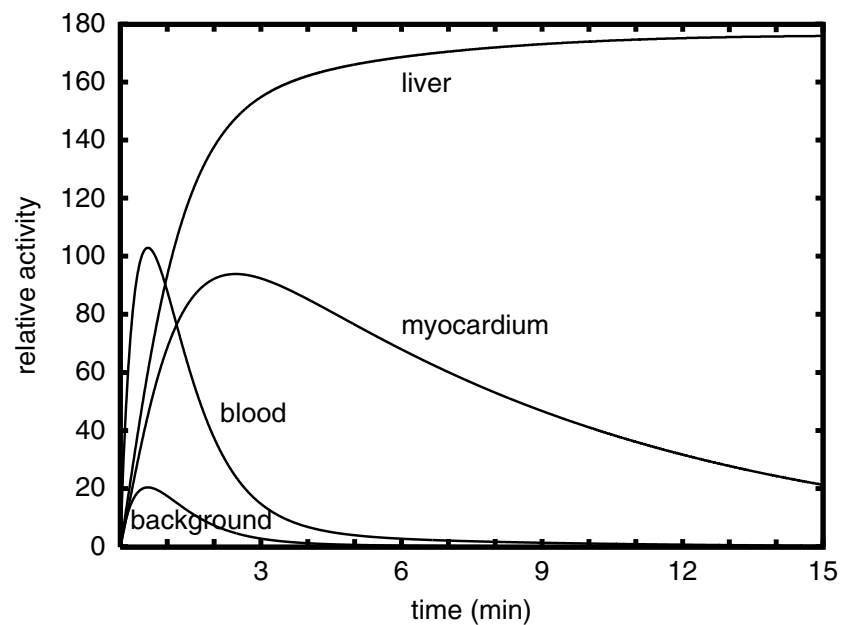

Fig. 2. Simulated time-activity curves, $A^{m}(t)$, for ${ }^{99 \mathrm{~m}} \mathrm{Tc}$-teboroxime.

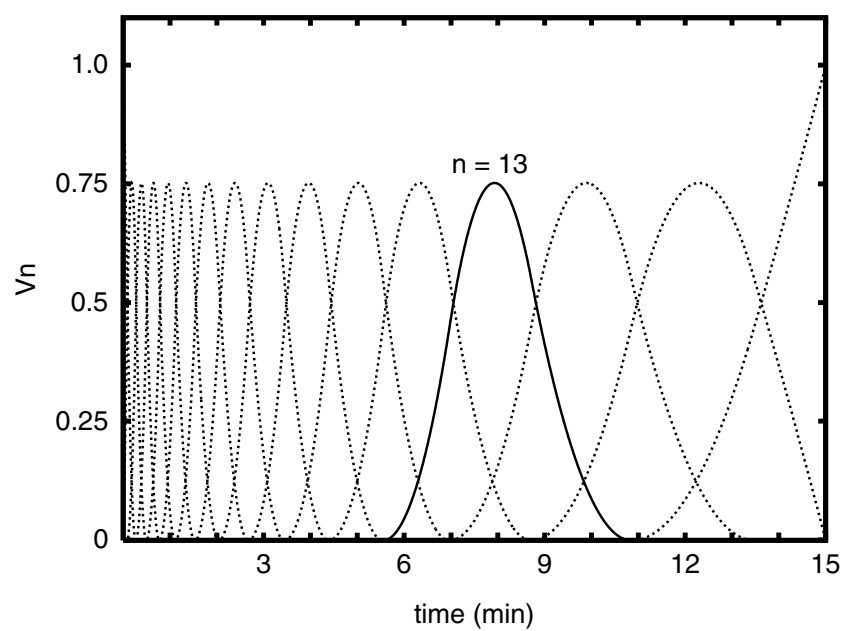

Fig. 3. Piecewise quadratic B-spline temporal basis functions, $V^{n}(t)$, used to model time-activity curves. The thirteenth spline is shown as a solid curve.

The projection space distribution of scatter from volume of interest $m$ was assumed to have known shape, $S_{i}^{m}(t)$, but unknown amplitude, $c_{m}$. Thus, the dynamic data model (2) was expressed as

$$
P_{i}(t)=\sum_{m=1}^{M}\left[D_{i}^{m}(t)+c_{m} S_{i}^{m}(t)\right] \sum_{n=1}^{N} a_{m n} V^{n}(t) .
$$

The model for the acquired projection data was obtained by integrating (8) over the $L=1800$ time intervals that spanned the data acquisition:

$$
p_{i l}=\int_{t_{l-1}}^{t_{l}} P_{i}(\tau) d \tau .
$$

The spline time-activity curve coefficients, $a_{m n}$, and scatter amplitudes, $c_{m}$, were jointly estimated with use of the LevenbergMarquardt algorithm [16] to minimize the sum of squared differences between the simulated and modeled projections:

$$
\chi^{2}=\sum_{i=1}^{I} \sum_{l=1}^{L}\left(p_{i l}^{*}-p_{i l}\right)^{2}
$$

where $p_{i l}^{*}$ were the simulated projections.

\section{RESULTS}

Fig. 4 shows spatial projection functions for unscattered events, $D_{i}^{m}(t)$, and scattered events, $S_{i}^{m}(t)$, for one period ( $T=1 \mathrm{~min}$ ). As expected, projections of the blood pool and myocardium overlapped extensively (Fig. 4a-d), and the liver had a spatial signature (Fig. 4e,f) that distinguished it clearly from the blood pool and myocardium. Of course, there was considerable overlap between projections of the background tissue (Fig. 4g,h) and the other volumes.

Fig. 5 shows unscattered and scattered components of dynamic projection data, i.e., $D_{i}^{m}(t) A^{m}(t)$ and $S_{i}^{m}(t) A^{m}(t)$, for the first $10 \mathrm{~min}$. The blood pool, myocardium, and liver had relatively distinct spatiotemporal signatures (Fig. 5a-f). However, projections of the background tissue (Fig. 5g,h) and blood pool overlapped temporally as well as spatially, because their time-activity curves had the same shape (Fig. 2).

Dynamic data acquisition and quantitative kinetic data analysis enabled joint estimation of scatter amplitudes and timeactivity curves by exploiting the fact that the scatter distribution from a volume of interest has the same temporal kinetics as unscattered activity from the volume [equation (8)]. For noiseless data, time-activity curve estimates closely fit the simulated curves (Table I) and scatter amplitude estimates ranged between $0.988-1.001$, compared to the simulated value of 1 .

For 500k noisy events, noise-to-signal ratios (NSRs) for the time-activity curves were $0.9-16 \%$ when scatter amplitudes were known, and worsened to 1.1-25\% when scatter amplitudes were jointly estimated (Table I). NSR changed by a factor of $\sim \sqrt{2}$ when the number of events changed by a factor of 2 , as expected. The blood pool time-activity curve had the worst NSR because of the large number of spatially overlapping events from the background tissue, which had the same kinetics as blood.

Reducing the background tissue amplitude by a factor of 2 , while maintaining overall counts at $500 \mathrm{k}$, improved blood NSR by a factor of 1.27 when jointly estimating scatter (Table II). We anticipate that the blood NSR will further improve when jointly estimating compartmental models for the myocardium and liver as proposed in [7], because of the additional temporal modeling constraint that the myocardial and liver time-activity curves are obtained by convolution with the blood curve.

\section{Future Directions}

We are working to fully implement the two proposed scatter models given by (5) and (6), and to use the models in conjunction with the dynamic projection data model given by (2). Methods described in [7] are being extended so that scatter 
(a)

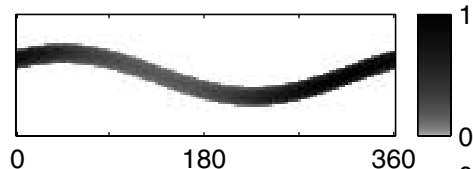

(b)

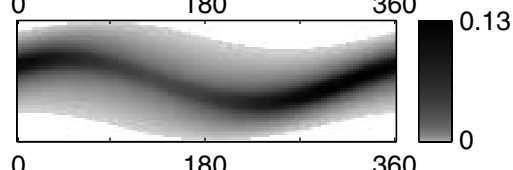

(c)

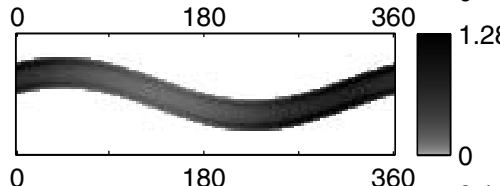

(d)

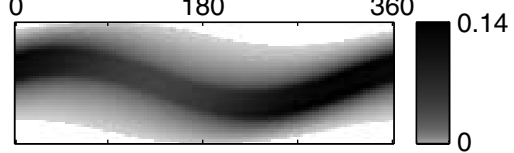

(e)

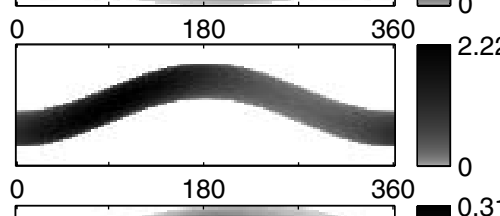

(f)

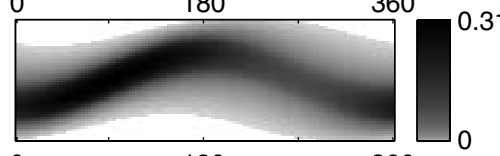

(g)
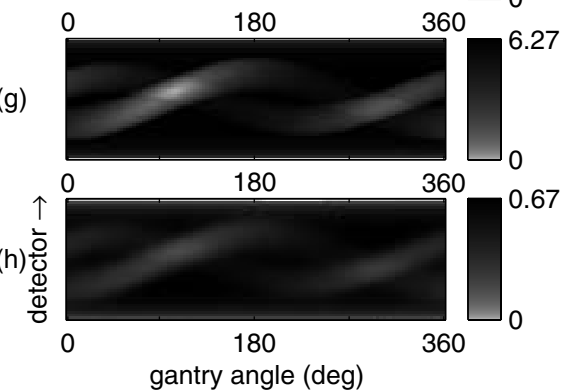

Fig. 4. One period of spatial projection functions. Unscattered events, $D_{i}^{m}(t)$, are shown for (a) blood pool, (c) myocardium, (e) liver, and (g) background tissue. Scattered events, $S_{i}^{m}(t)$, are shown for (b) blood pool, (d) myocardium, (f) liver, and (h) background tissue. Numbers labeling color bars reflect relative geometric sensitivity. (a)
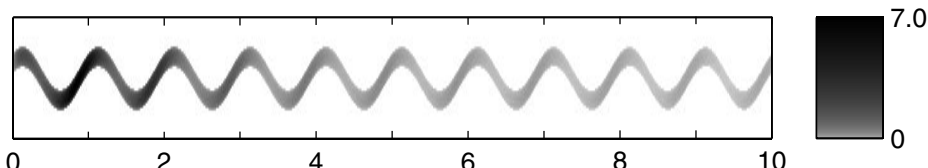

(b)
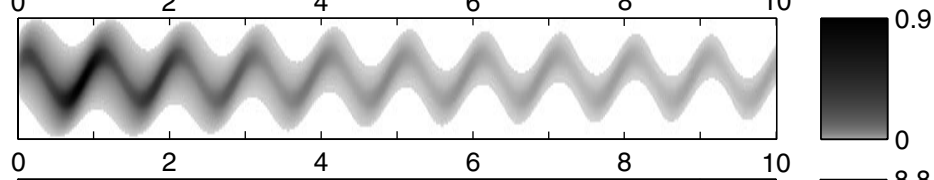

(c)
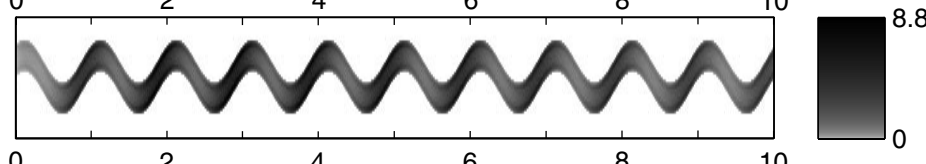

(d)
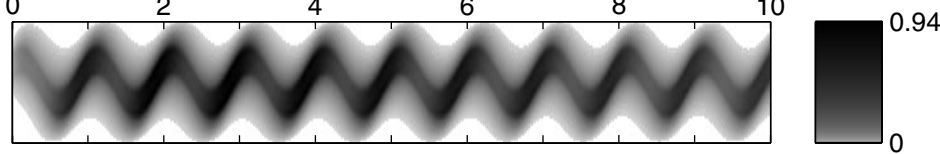

(e)
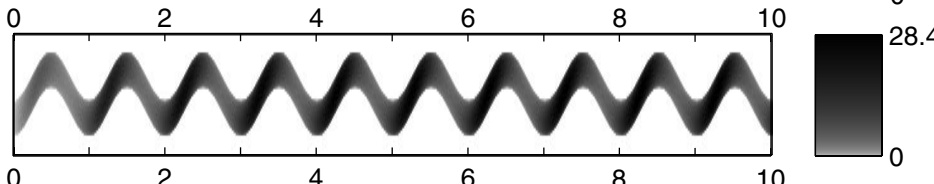

(f)
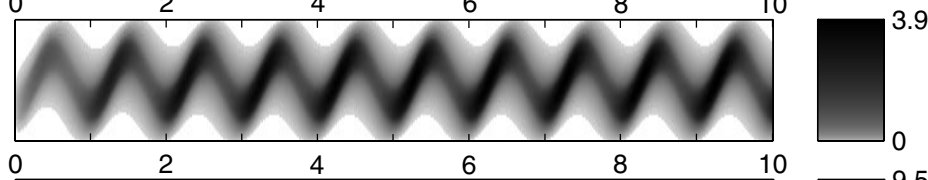

(g)
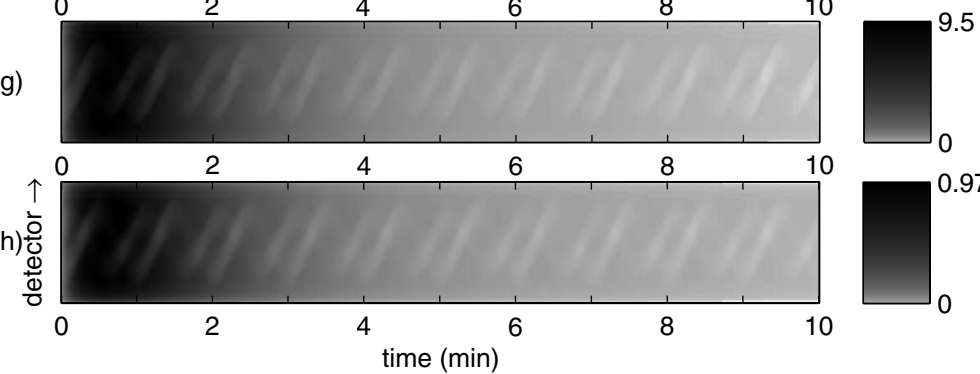

Fig. 5. Unscattered and scattered components of simulated noiseless, attenuated dynamic ${ }^{99}$ Tc-teboroxime projection data. Dynamic unscattered events, $D_{i}^{m}(t) A^{m}(t)$, are shown for (a) blood pool, (c) myocardium, (e) liver, and (g) background tissue. Dynamic scattered events, $S_{i}^{m}(t) A^{m}(t)$, are shown for (b) blood pool, (d) myocardium, (f) liver, and (h) background tissue. Numbers labeling color bars are expected numbers of events in sinogram bins for a dynamic study in which 500,000 total events are detected. parameters, $b_{r m}$ in (5) or $c_{r m}$ in (6), can be jointly estimated with linear and nonlinear kinetic parameters for time-activity curves, $A^{m}(t)$ in (2). We will then compare kinetic parameter estimates obtained with the proposed scatter models and a scatter model determined by Monte Carlo simulation.

\section{ACKNOWLEDGMENT}

This work was supported by the U.S. Department of Health and Human Services under grants R01-HL50663 and R01EB00121 and by the Director, Office of Science, Office of Biological and Environmental Research, Medical Sciences Division of the U.S. Department of Energy under contract DE-AC0376SF00098. This work was developed in part with the use of resources at the U.S. Department of Energy National Energy Research Scientific Computing (NERSC) Center.

\section{REFERENCES}

[1] G. T. Gullberg, R. H. Huesman, S. G. Ross, E. V. R. Di Bella, G. L. Zeng, B. W. Reutter, P. E. Christian, and S. A. Foresti, "Dynamic cardiac single-photon emission computed tomography," in Nuclear Cardiology: State of the Art and Future Directions, B. L. Zaret and G. A. Beller, Eds. St. Louis: Mosby Inc., 1999, ch. 11, pp. 137-187.

[2] E. V. R. Di Bella, S. G. Ross, D. J. Kadrmas, H. S. Khare, P. E. Christian, S. McJames, and G. T. Gullberg, "Compartmental modeling of technetium-99m-labeled teboroxime with dynamic single-photon emission computed tomography: Comparison with static thallium-201 in a canine model," Invest. Radiol., vol. 36, no. 3, pp. 178-185, 2001.

[3] B. W. Reutter, G. T. Gullberg, and R. H. Huesman, "Effects of scatter modeling on time-activity curves estimated directly from dynamic SPECT projections," in 2003 IEEE Nuclear Science Symposium and Medical Imaging Conference Record, S. Metzler, Ed., 2004, pp. M10-289/1-5.

[4] R. H. Huesman, B. W. Reutter, G. L. Zeng, and G. T. Gullberg, "Kinetic parameter estimation from SPECT cone-beam projection measurements," Phys. Med. Biol., vol. 43, no. 4, pp. 973-982, 1998.

[5] B. W. Reutter, G. T. Gullberg, and R. H. Huesman, "Direct least- 
TABLE I

ROOT MEAN SQUARE (RMS) ERRORS FOR TIME-ACTIVITY CURVES ESTIMATED FROM NOISELESS DATA, AND EFFECTS OF JOINT ESTIMATION OF SCATTER

AMPLITUDES ON NOISE-TO-SIGNAL RATIOS (NSRS) FOR TIME-ACTIVITY CURVES ESTIMATED FROM 1M, 500K, OR 250K NOISY EVENTS. THE BACKGROUND TISSUE TIME-ACTIVITY CURVE AMPLITUDE WAS 0.2 TIMES THAT OF THE BLOOD POOL (FIG. 2). RMS ERROR WAS CALCULATED AS THE RMS DIFFERENCE BETWEEN THE ESTIMATED AND SIMULATED TIME-ACTIVITY CURVES, NORMALIZED BY THE RMS VALUE FOR THE SIMULATED CURVE AND EXPRESSED AS A PERCENTAGE. NSR WAS CALCULATED AS THE ROOT MEAN VALUE (ACROSS TIME) OF EXPECTED SQUARED DIFFERENCES BETWEEN TIME-ACTIVITY CURVES ESTIMATED FROM NOISY AND NOISELESS DATA, NORMALIZED BY THE RMS VALUE FOR THE NOISELESS CURVE AND EXPRESSED AS A PERCENTAGE [6]

\begin{tabular}{|c|c|c|c|c|c|c|c|}
\hline & \multirow{3}{*}{$\begin{array}{l}\text { RMS err } \\
\text { noiseless } \\
(\%)\end{array}$} & \multicolumn{3}{|c|}{ scatter amplitude known } & \multicolumn{3}{|c|}{ scatter amplitude estimated } \\
\hline & & NSR & NSR & NSR & NSR & NSR & NSR \\
\hline & & $\begin{array}{c}1 \mathrm{M} \text { cts } \\
(\%)\end{array}$ & $\begin{array}{c}500 \mathrm{k} \text { cts } \\
(\%)\end{array}$ & $\begin{array}{c}250 \mathrm{k} \mathrm{cts} \\
(\%)\end{array}$ & $\begin{array}{c}1 \mathrm{M} \text { cts } \\
(\%)\end{array}$ & $\begin{array}{c}500 \mathrm{k} \text { cts } \\
(\%)\end{array}$ & $\begin{array}{c}250 \mathrm{k} \mathrm{cts} \\
(\%)\end{array}$ \\
\hline blood pool & 0.6 & 11.3 & 15.9 & 22.5 & 17.3 & 24.5 & 34.7 \\
\hline myocardium & 0.2 & 3.8 & 5.4 & 7.7 & 4.7 & 6.6 & 9.4 \\
\hline liver & 0.03 & 0.6 & 0.9 & 1.2 & 0.8 & 1.1 & 1.6 \\
\hline background & 0.5 & 1.0 & 1.3 & 1.9 & 2.7 & 3.8 & 5.3 \\
\hline
\end{tabular}

TABLE II

SAME QUANTITIES AS IN TABLE I, FOR THE CASE WHERE THE BACKGROUND TISSUE TIME-ACTIVITY CURVE AMPLITUDE WAS 0.1 TIMES THAT OF THE BLOOD POOL.

\begin{tabular}{c|c|ccc|ccc}
\hline & & \multicolumn{4}{|c|}{ scatter amplitude known } & \multicolumn{3}{c}{ scatter amplitude estimated } \\
\cline { 3 - 8 } & RMS err & NSR & NSR & NSR & NSR & NSR & NSR \\
& noiseless & $1 \mathrm{M}$ cts & $500 \mathrm{k}$ cts & 250k cts & $1 \mathrm{M}$ cts & $500 \mathrm{k}$ cts & $250 \mathrm{k} \mathrm{cts}$ \\
& $(\%)$ & $(\%)$ & $(\%)$ & $(\%)$ & $(\%)$ & $(\%)$ & $(\%)$ \\
\hline blood pool & 0.6 & 9.3 & 13.1 & 18.6 & 13.7 & 19.3 & 27.3 \\
\hline myocardium & 0.1 & 3.1 & 4.4 & 6.3 & 3.8 & 5.4 & 7.6 \\
\hline liver & 0.02 & 0.5 & 0.8 & 1.1 & 0.7 & 1.0 & 1.4 \\
\hline background & 0.5 & 1.4 & 2.0 & 2.9 & 3.7 & 5.3 & 7.4 \\
\hline
\end{tabular}

squares estimation of spatiotemporal distributions from dynamic SPECT projections using a spatial segmentation and temporal B-splines," IEEE Trans. Med. Imag., vol. 19, no. 5, pp. 434-450, 2000.

[6] B. W. Reutter, R. H. Huesman, and G. T. Gullberg, "Effects of temporal modelling on the statistical uncertainty of spatiotemporal distributions estimated directly from dynamic SPECT projections," Phys. Med. Biol., vol. 47, no. 15 , pp. 2673-2683, 2002.

[7] B. W. Reutter, G. T. Gullberg, and R. H. Huesman, "Fully 4-D direct joint estimation of compartmental models and blood input function from dynamic SPECT projections," in Proceedings of the VIIth International Conference on Fully $3 D$ Reconstruction in Radiology and Nuclear Medicine, Y. Bizais, Ed., 2003, pp. Mo-PM3-1/1-4.

[8] I. Buvat and I. Castiglioni, "Monte Carlo simulations in SPET and PET," Q. J. Nucl. Med., vol. 46, no. 1, pp. 48-61, 2002.

[9] I. Laurette, G. L. Zeng, A. Welch, P. E. Christian, and G. T. Gullberg, "A three-dimensional ray-driven attenuation, scatter and geometric response correction technique for SPECT in inhomogeneous media," Phys. Med. Biol., vol. 45, no. 11, pp. 3459-3480, 2000.

[10] E. C. Frey and B. M. W. Tsui, "A new method for modeling the spatially-variant, object-dependent scatter response function in SPECT," in 1996 IEEE Nuclear Science Symposium and Medical Imaging Conference Record, A. Del Guerra, Ed., 1997, pp. 1082-1086.
[11] H. E. Johns and J. R. Cunningham, The Physics of Radiology, 4th ed. Springfield, IL: Thomas, 1983.

[12] R. H. Bartels, J. C. Beatty, and B. A. Barsky, An Introduction to Splines for Use in Computer Graphics and Geometric Modeling. Los Altos, CA: Morgan Kaufmann Publishers, Inc., 1987.

[13] R. K. Narra, T. Feld, and A. D. Nunn, "Absorbed radiation dose to humans from technetium-99m-teboroxime," J. Nucl. Med., vol. 33, no. 1, pp. 8893, 1992.

[14] R. H. Huesman, G. T. Gullberg, W. L. Greenberg, and T. F. Budinger, "RECLBL library users manual - Donner algorithms for reconstruction tomography," Lawrence Berkeley Laboratory, Publication PUB-214, 1977.

[15] A. Welch, G. T. Gullberg, P. E. Christian, F. L. Datz, and H. T. Morgan, "A transmission-map-based scatter correction technique for SPECT in inhomogeneous media," Med. Phys., vol. 22, no. 10, pp. 1627-1635, 1995.

[16] W. H. Press, S. A. Teukolsky, W. T. Vetterling, and B. P. Flannery, Numerical Recipes in C: The Art of Scientific Computing, 2nd ed. Cambridge: Cambridge University Press, 1992. 